\title{
Design of refractive laser beam shapers to generate complex irradiance profiles
}

\author{
Meijie Li, ${ }^{* a}$ Youri Meuret ${ }^{a, b}$ Fabian Duerr, ${ }^{a}$ Michael Vervaeke,${ }^{a}$ Hugo Thienpont ${ }^{a}$ \\ ${ }^{a}$ Vrije Universiteit Brussel, Brussels Photonics Team, Pleinlaan 2, Brussels, Belgium, B-1050; \\ ${ }^{b}$ KU Leuven, Faculteit Industriële Ingenieurswetenschappen, Light \& Lighting Laboratory, \\ Gebroeders De Smetstraat 1, Gent, Belgium, B-9000
}

\begin{abstract}
A Gaussian laser beam is reshaped to have specific irradiance distributions in many applications in order to ensure optimal system performance. Refractive optics are commonly used for laser beam shaping. A refractive laser beam shaper is typically formed by either two plano-aspheric lenses or by one thick lens with two aspherical surfaces. Ray mapping is a general optical design technique to design refractive beam shapers based on geometric optics. This design technique in principle allows to generate any rotational-symmetric irradiance profile, yet in literature ray mapping is mainly developed to transform a Gaussian irradiance profile to a uniform profile. For more complex profiles especially with low intensity in the inner region, like a Dark Hollow Gaussian (DHG) irradiance profile, ray mapping technique is not directly applicable in practice. In order to these complex profiles, the numerical effort of calculating the aspherical surface points and fitting a surface with sufficient accuracy increases considerably. In this work we evaluate different sampling approaches and surface fitting methods. This allows us to propose and demonstrate a comprehensive numerical approach to efficiently design refractive laser beam shapers to generate rotational-symmetric collimated beams with a complex irradiance profile. Ray tracing analysis for several complex irradiance profiles demonstrates excellent performance of the designed lenses and the versatility of our design procedure.
\end{abstract}

Keywords: refractive laser beam shaping, complex irradiance distributions, free-form surface construction, splines, polynomials

\section{INTRODUCTION}

Laser beam shaping is widely used in many industrial and medical applications. In most cases, a laser beam with a Gaussian irradiance profile is reshaped to have a uniform output profile, usually called a flat-top profile. In some applications, like optical trapping, ${ }^{1}$ atom guiding ${ }^{2}$ and laser additive manufacturing, a complex irradiance profile is required in order to ensure optimal system performance. One example of such a complex irradiance profile is a Dark Hollow Gaussian (DHG) irradiance profile. There are various proposed techniques for laser beam shaping, especially to generate rotational symmetric flat-top irradiance profiles. The simplest technique is either to truncate an expanded Gaussian beam ${ }^{3}$ or to use an apodized absorption filter. ${ }^{4}$ Such a technique allows to generate uniform intensity patterns but is rather energy-inefficient. Typical energy-efficient approaches include refractive ${ }^{5}$ or reflective systems, ${ }^{6}$ diffractive elements, ${ }^{7}$ holograms ${ }^{8}$ and microlens arrays. ${ }^{9}$ Among these techniques, refractive laser beam shapers are commonly used due to their high optical efficiency and simple structure. $^{10}$

A typical refractive laser beam shaper consists either two plano-aspheric lenses or one single thick lens with two aspherical surfaces. The first aspherical surface redistributes the beam irradiance while the second aspherical surface recollimates the beam. Four general configurations are shown in Fig. 1. Compared with the Kepler type at top, the Galilean type at bottom is more compact. For high power laser applications, the Galilean type should be used because it does not have the internal beam focus.

The general optical design approach of such refractive beam shapers is based on ray mapping within the framework of geometric optics. Most of the literature focusses on the problem of transforming a collimated

*E-mail: mli@b-phot.org; Tel: +32 262936 62; Fax: +32 26293450

Optical Modelling and Design III, edited by Frank Wyrowski, John T. Sheridan, Jani Tervo,

Youri Meuret, Proc. of SPIE Vol. 9131, 913105 - (c) 2014 SPIE

CCC code: $0277-786 X / 14 / \$ 18 \cdot$ doi: $10.1117 / 12.2052353$

Proc. of SPIE Vol. $9131913105-1$ 


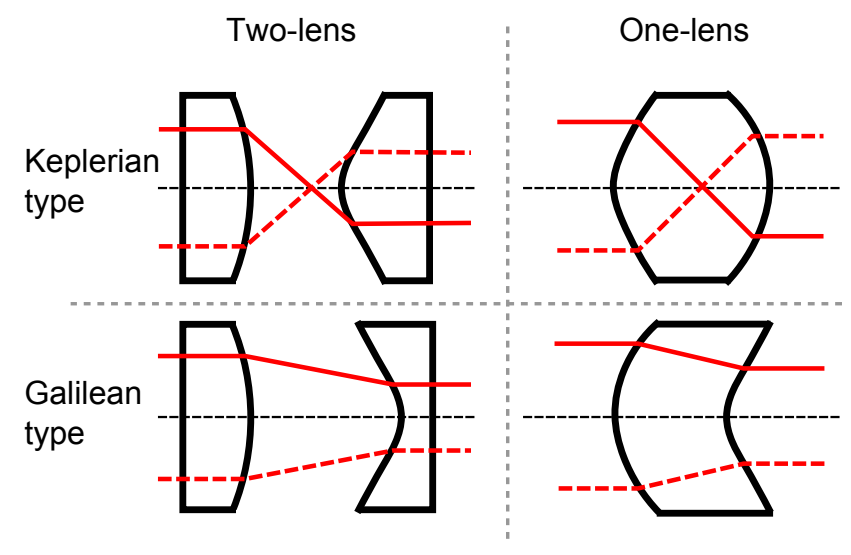

Figure 1. Refractive laser beam shapers can be divided in 4 general configurations.

Gaussian beam to a collimated beam with a uniform irradiance profile. The problem was initially proposed by Frieden in $1965 .{ }^{11}$ Then Kreuzer patented a design procedure in $1969,{ }^{12}$ which is still widely used today. The ray mapping technique was further investigated and developed by Rhodes ${ }^{13}$ and Hoffnagle. ${ }^{5,14,15}$ Additional research led to simplification of the practical design steps ${ }^{16}$ and optimization of the design algorithm for better performance. ${ }^{17}$

With the ray mapping technique, it is in theory possible to generate any irradiance profile. However published works mainly focus on flat-top profile generation and do not describe the practical steps on how to generate more complex profiles. For complex irradiance profiles especially with low intensity in the inner region, it is difficult to derive a solution analytically and the available numerical design approach ${ }^{16}$ to generate elementary profiles is not directly applicable to obtain more complex profiles (e.g. a DHG profile). Based on the ray mapping technique, this work presents an efficient numerical approach to design refractive laser beam shapers for various complex irradiance profiles.

The design of a refractive beam shaper based on geometric ray mapping can typically be divided into four steps. In order to introduce these four steps, the conventional design approach for generating simple irradiance profiles is firstly reviewed in Sec. 2. In Sec. 3, our new proposed approach to obtain complex irradiance profiles is described. There are two essential design steps involved in our approach. In the first step to calculate the mapping function between the radial positions on the two aspherical surfaces, an alternative sampling method is proposed to work more efficiently compared with conventional sampling method. In the last step of constructing the designed surfaces, two methods are investigated. One method is polynomial fitting and the other one is spline fitting which describes the surface profile by piecewise low degree polynomials. In Sec. 4, ray tracing results for different complex irradiance profiles are presented and analyzed. Taking a DHG profile as an example, the efficiency of two different sampling methods are compared with each other, highlighting the advantage of the alternative sampling method we propose. Additionally, the accuracy of two different surface construction methods is discussed and compared. In the end, additional simulation results for two other complex irradiance profiles are presented, underlining the versatility of our design procedure. Finally, in Sec. 5, conclusions are drawn and an outlook is given.

\section{CONVENTIONAL APPROACH FOR GENERATING SIMPLE IRRADIANCE PROFILES}

Conventional approach to design refractive beam shapers based on ray mapping technique is well developed to generate simple irradiance profiles, such as a flat-top profile. This section describes the conventional approach following four design steps in details. The one-lens Kepler-type configuration (see Fig. 2) is used for all designs in this article. It should be noticed that the presented approach can be easily adapted to design other configurations in Fig. 1, by modifying only the slope function Eq. (7) in Step 2. 


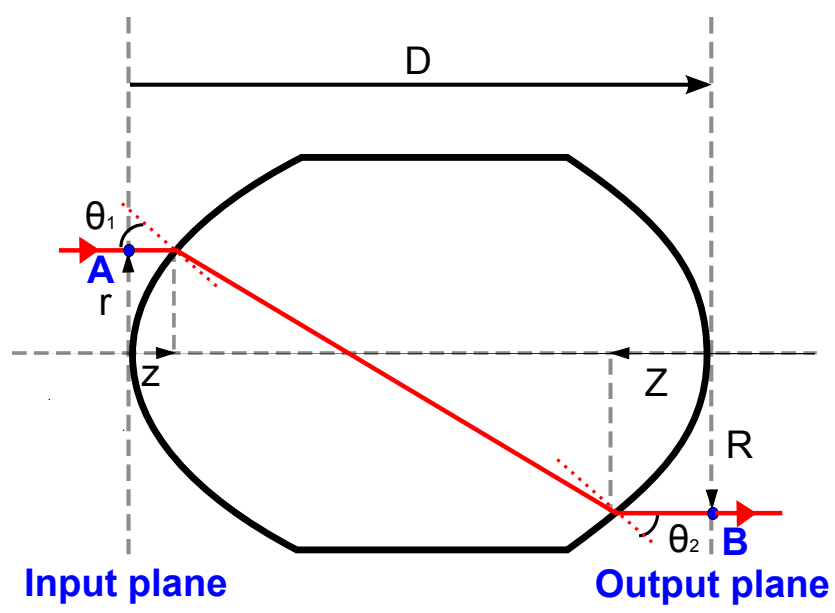

Figure 2. Illustration of the one-lens Kepler-type configuration that transforms a collimated input beam to a collimated output beam: an arbitrary ray intersects the input plane at radius $r$ and arrives at the output plane at radius $R$; $z$ and $Z$ describe the sag values of the first and second aspherical surfaces; $D$ is the thickness of the lens.

\section{Step 1: Calculating the mapping function}

The mapping function is the mapping relationship between the radial positions on the two aspherical surfaces $r$ and $R$. Based on the law of conservation of energy, the power at the input plane is equal to the the power at the output plane assuming there is no energy loss through the lens system. In order to map every radial coordinate $r$ to $R$, the power inside each circle of the input radial coordinate $r$ should be the same as the power at the inside of each circle of the output radial coordinate $R$.

Both the input and output irradiance profiles are rotationally symmetric, and can be described by functions $F_{\text {in }}(r)$ and $F_{\text {out }}(R)$. The encircled power within $r$ at the input plane is a function of $r$, given by the integral function $G_{i n}(r)$ :

$$
G_{i n}(r)=2 \pi \int_{0}^{r} F_{i n}(x) \cdot x \cdot d x .
$$

Similarly at the output plane, the function $G_{\text {out }}(R)$ calculates the encircled power within $R$ as

$$
G_{\text {out }}(R)=2 \pi \int_{0}^{R} F_{\text {out }}(x) \cdot x \cdot d x .
$$

Then the total power $P$ is directly sampled into equal power bins and for each power bin the corresponding radial position on the first aspherical surface $r$ and on the second aspherical surface $R$ are calculated accordingly. We call this conventional sampling method Sampling $\boldsymbol{P}$. It works as follows:

The total input and output power are both normalized to 1 or $100 \%$. Firstly the maximum radii at the input and output planes $r_{\max }$ and $R_{\max }$ should be chosen properly so that sufficient power $P_{\max }$ (e.g. 99.9\%) can be transferred from the input to the output. Then the total power $P_{\max }$ is sampled by equal bins. Supposing the sampling number is $N$, the sampling index $i$ becomes $\{1,2, \cdots, N\}$. There are in total $(N-1)$ bins. For each bin the encircled power is then given as

$$
P_{i}=\left\{0, \frac{99.9 \%}{N-1}, 2 \times \frac{99.9 \%}{N-1}, \cdots, 99.9 \%\right\} .
$$

For each power bin, the corresponding radii at the input plane and output plane $r_{i}$ and $R_{i}$ can be derived from the inverse functions of $G_{i n}(r)$ and $G_{\text {out }}(R)$ :

$$
\begin{aligned}
r_{i} & =G_{\text {in }}^{-1}\left(P_{i}\right), \\
R_{i} & =G_{\text {out }}^{-1}\left(P_{i}\right) .
\end{aligned}
$$

In this way, $r$ is mapped to $R$ and the generated data pairs $\left\{r_{i}, R_{i}\right\}$ are used for further calculation. 


\section{Step 2: Calculating the surface slopes}

In Fig. 2, the slopes $v_{i}$ and $V_{i}$ are of two aspheric lens profiles are defined as

$$
v_{i}=\tan \theta_{1}, \quad V_{i}=-\tan \theta_{2}
$$

Due to the fact that both input and output beams are collimated, it follows from symmetry that the angles $\theta_{1}$ and $\theta_{2}$ are equal and as a result, $v_{i}=-V_{i}$.

By applying Snells law at each aspherical surface and keeping the optical path length (OPL) between the input plane and the output plane constant, the slope values $v_{i}$ and $V_{i}$ can be described as a function of $r_{i}, R_{i}$, the thickness of the lens $D$ and the refractive index of the lenses $n_{2} \cdot{ }^{16}$ The local slope values at every radial position are calculated by

$$
v_{i}=-V_{i}=\frac{R_{i}+r_{i}}{\sqrt{\left(\frac{1}{n_{2}^{2}}-1\right)\left(R_{i}+r_{i}\right)^{2}+D^{2}\left(\frac{1}{n_{2}}-1\right)^{2}}} .
$$

\section{Step 3: Calculating the surface sags}

After the calculation of the local slopes at each surface, the surface sags $z_{i}$ and $Z_{i}$ can be calculated by integrating the slopes numerically. The specific numerical integration method should be selected carefully in order to minimize the numerical calculation error. In this article, the trapezoidal numerical integration is used, available within MATLAB ${ }^{18}$ via the command "TRAPZ".

\section{Step 4: Constructing the aspherical surfaces}

In order to evaluate the optical performance of the beam shaper it is necessary to construct smooth surfaces from the calculated discrete sets of sag and/or slope values. Mostly used surface constructing method is Polynomial Fitting. With polynomial fitting an analytical polynomial function is applied to describe the designed surfaces. To generate a flat-top profile, it is sufficient to use a polynomial function with only even exponents: ${ }^{16}$

$$
z(r)=A_{1} r^{2}+A_{2} r^{4}+\cdots+A_{n} r^{2 n} .
$$

The coefficients $\left\{A_{1}, A_{2}, \cdots, A_{n}\right\}$ can be determined by either fitting the polynomial function $z(r)$ to the calculated sags or by fitting its first derivative $z^{\prime}(r)$ to the calculated slopes. In this article, the polynomial function is fitted to the calculated sags in MATLAB ${ }^{18}$ using the command "POLYFITN". This algorithm calculates the coefficients of the polynomial function that fits the data set best by minimizing the least-squares.

\section{NEW PROPOSED APPROACH FOR GENERATING COMPLEX IRRADIANCE PROFILES}

The design approach introduced in Sec. 2 does not work properly for generating complex irradiance profiles especially with low intensity in the inner region. Following the same four design steps, our new proposed approach is given in this section. It can easily be followed to obtain complex irradiance profiles, like a DHG profile.

\section{Step 1: Calculating the mapping function}

In order to generate complex irradiance patternss, we propose an equidistant sampling of the output radial coordinate $R$ as an efficient alternative of sampling $P$. More precisely the complexity of the input and output irradiance profiles has to be compared properly and the radial coordinate at the plane with more complex profile should be sampled. As the input beam is always a laser beam with Gaussian distribution, the desired output profile (e.g. a DHG profile) is usually more complex than the input Gaussian profile, which means the output radial coordinate $R$ should be sampled. So we name this alternative sampling method as Sampling $\boldsymbol{R}$.

In this case we work as follows: 
a) A maximum value for $R$ has to be chosen large enough to transmit sufficient power $P$ (e.g. $P_{\max }=99.9 \%$ ). The value $R_{\max }$ should be verified to ensure a feasible aperture size for the designed lens. $R_{\max }$ is calculated by

$$
R_{\max }=G_{\text {out }}^{-1}\left(P_{\max }\right) .
$$

b) $R_{\max }$ is sampled equidistantly by sufficient number of points $N$. Similarly as before, the sampling index $i$ is $\{1,2, \cdots, N\}$. Then

$$
R_{i}=\left\{0, \frac{R_{\max }}{N-1}, 2 \times \frac{R_{\max }}{N-1}, \cdots, R_{\max }\right\} .
$$

c) The encircled power within $R_{i}$ is calculated by

$$
P_{i}=G_{\text {out }}\left(R_{i}\right) .
$$

d) The corresponding $r_{i}$ at the input plane is calculated by

$$
r_{i}=G_{i n}^{-1}\left(P_{i}\right),
$$

The calculated data pairs $\left\{r_{i}, R_{i}\right\}$ provide the mapping relationship between $r$ and $R$.

\section{Step 2: Calculating the surface slopes}

It is the same as Step $\mathbf{2}$ in Sec. 2.

\section{Step 3: Calculating the surface sags}

It is the same as Step 3 in Sec. 2.

\section{Step 4: Constructing the aspherical surfaces}

For complex irradiance profiles, we have found that a polynomial function with only even exponents is not adequate, no matter how many coefficients are used. Instead a polynomial function with both even and odd exponents should be used:

$$
z(r)=A_{1} r+A_{2} r^{2}+\cdots+A_{n} r^{n} .
$$

Here it should be noticed that $r$ is always positive and the pattern is rotationally symmetric. The coefficients $\left\{A_{1}, A_{2}, \cdots, A_{n}\right\}$ can be determined in the same way as in last section.

In addition to polynomial fitting, Spline Fitting method is investigated as an alternative surface constructing method. Spline fitting uses piecewise low order polynomial functions to match both calculated sag and slope values in order to describe the surface profile. ${ }^{19}$ We have used a continuous piecewise cubic spline to construct the designed aspherical surfaces in the optical ray tracing software ASAP ${ }^{20}$ with the "SPLINE" command.

\section{RAY TRACING RESULTS AND DISCUSSION}

Our designed refractive beam shapers are evaluated by ray tracing in ASAP and the simulation results are presented and discussed in this section.

The input and output irradiance profiles of our example to transform a Gaussian profile to a DHG profile are shown in Fig. 3. The input beam has a circular Gaussian irradiance profile. The irradiance of a Gaussian profile $I_{G}$ is expressed by

$$
I_{G}(r)=I_{a} \times \exp \left[-2\left(\frac{r}{w_{G}}\right)^{2}\right],
$$

where $r$ is the radial coordinate, $I_{a}$ is the maximum (max.) irradiance at $r=0$, and $w_{G}$ is the beam waist. The irradiance at the beam waist is equal to $I_{a} \times e^{-2}$. In our example, $I_{a}$ is calculated by normalizing the total 
power to 1 , and $w_{G}$ is $2.366 \mathrm{~mm}$. The output beam has a DHG irradiance profile, so the beam can be described by Hollow Gaussian Beams (HGBs). ${ }^{21}$ Then the irradiance $I_{D H G}$ is calculated by

$$
I_{D H G}(r)=I_{b} \times\left(\frac{r^{2}}{w_{D H G}^{2}}\right)^{2 n} \times \exp \left(-2 \frac{r^{2}}{w_{D H G}^{2}}\right), \quad n=0,1,2, \ldots
$$

where $I_{b}$ is a constant, $n$ is the order of the HGB and $r$ is the radial coordinate. In the case $n$ is equal to 0 , the DHG is identical to the fundamental Gaussian with beam waist $w_{D H G}$. As energy is conserved, $I_{b}$ is calculated by normalizing the total power also to $1 . n$ is chosen as 1 , and $w_{D H G}$ is $2 \mathrm{~mm}$.

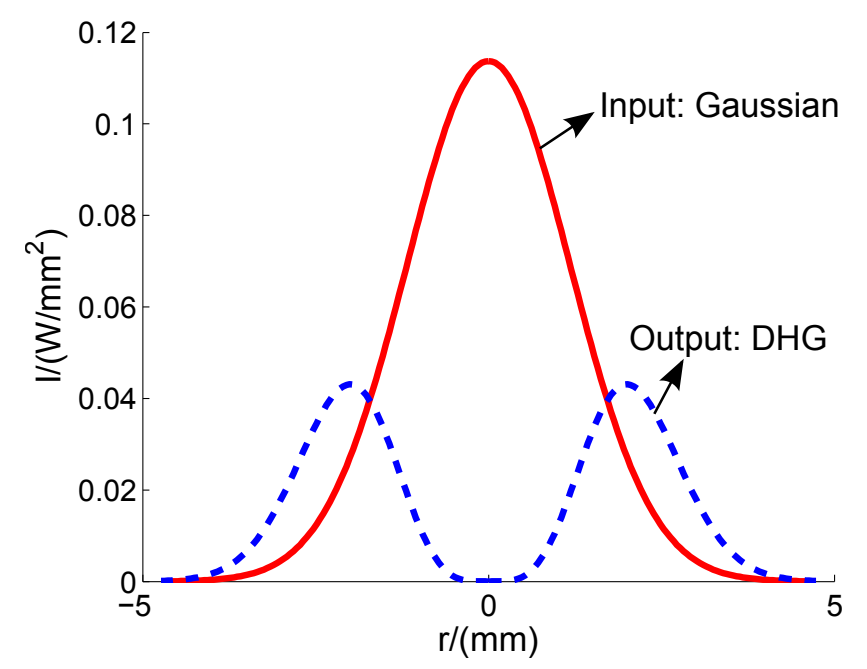

Figure 3. Irradiance profiles: input Gaussian profile and output DHG profile. $r$ is the radial coordinate and $I$ is the irradiance.

\subsection{Ray tracing results for designs obtained with different sampling methods}

Two sampling methods have been introduced in the first design step of calculating the mapping function: Sampling $\boldsymbol{P}$ for the conventional approach and Sampling $\boldsymbol{R}$ for the new proposed approach. In order to evaluate these two sampling methods, we have used both methods with different sampling numbers to design beam shapers that generate the same DHG profile. After constructing the calculated aspherical surfaces by spline fitting, both designs are simulated in ASAP and ray tracing results are compared.

With sampling $P$ method, several beam shapers are designed to generate the DHG output profile by using different numbers of sampling points. For each design, a grid of $2001 \times 2001$ rays has been traced through the lens system with ASAP to generate the output intensity profile directly after the second lens. A comparison of the detected output profiles for different designs is given in Fig. 4. In order to match the required DHG profile, the number of sampling points $N$ has to be as large as 10000. With less sampling points, the output profile departures from the expected DHG profile especially for the center part. With low number of sampling points, the center part can not be sampled adequately due to its low or zero-intensity.

The ray tracing result for a design with sampling $R$ by only 100 sampling points is shown in Fig. 5 . It clearly shows that 100 sampling points are already sufficient, which is much less than when we sample the encircled power $P$. With less sampling points, the numerical efforts to calculate the lens profiles and the simulation time to evaluate the designed beam shaper can be drastically reduced, as we will explain in more details in the next section. This finding highlights the benefits of the sampling $R$ method to efficiently generate complex irradiance profiles.

\subsection{Ray tracing results for designs obtained with different surface construction methods}

We consider two possible ways to construct surfaces from the calculated surface sages (and slopes): polynomial fitting and spline fitting. Designs obtained with these two methods are simulated in ASAP. For each simulation, 


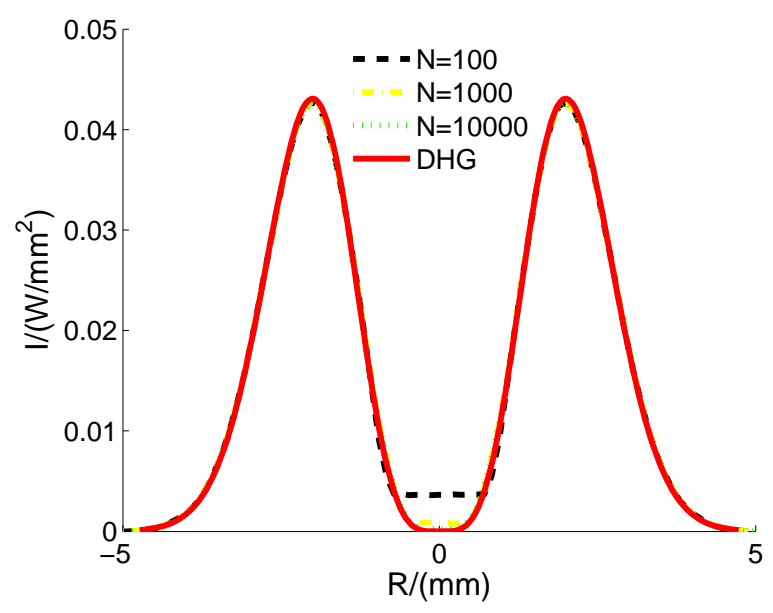

Figure 4. Ray tracing results for designs obtained with sampling $P$ method for different number $N$ of sampling points: the shown simulation results (dashed lines) in comparison with the required DHG profile (solid line) reveal the high sampling rate that is necessary to achieve good agreement of the intensities especially in the central region.

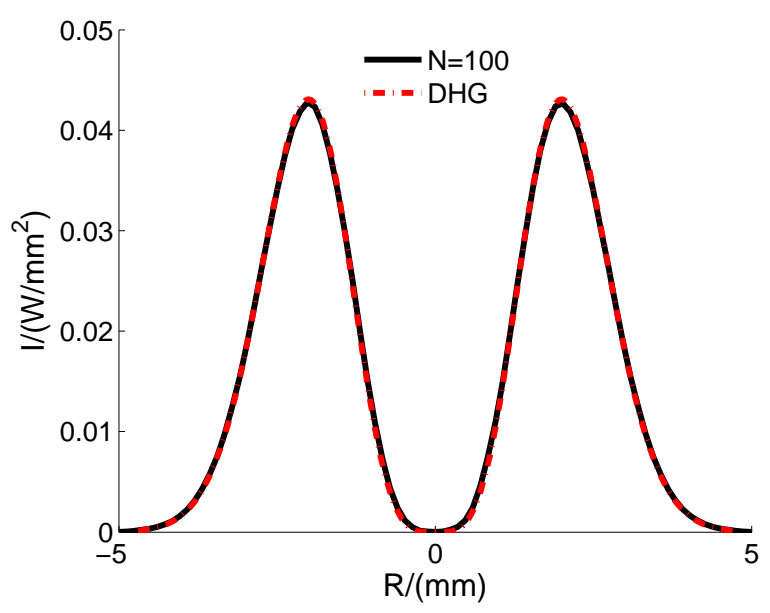

Figure 5. Ray tracing results for designs obtained with sampling $R$ method for a moderate number of only 100 sampling points: the simulated DHG irradiance profile (solid line) matches the required DHG output profile (dashed line) very well.

a grid of $2001 \times 2001$ rays is traced and evaluated this time not only at the plane directly after the second lens, but also at planes with certain distances from the second lens along the $z$ axis. In this way, it is possible to verify how well the generated output profile is maintained after propagation, which is a good indication on the collimation quality of the output beam.

For the method of polynomial fitting, a polynomial function in Eq. (13) with 21 coefficients is used. Ray tracing results for the design with polynomial fitting are presented in Fig. 6. The solid red line is the required DHG profile, whereas the different dashed lines correspond to the simulation results for the output profile after certain propagation distances along the $z$ axis. For $z=0.1 \mathrm{~mm}$, the output profile corresponds well to the expected DHG profile. The irradiance profile starts to deviate from the DHG profile when it propagates over distances beyond $100 \mathrm{~mm}$, and after $1000 \mathrm{~mm}$, the difference is very clear.

Figure 7 shows the simulation results for the design obtained with spline fitting. In this case the irradiance profile agrees well with the required DHG profile over the complete propagation trajectory which proves perfect collimation of the output beam. It should be noticed that the simulation time for surfaces constructed by spline 


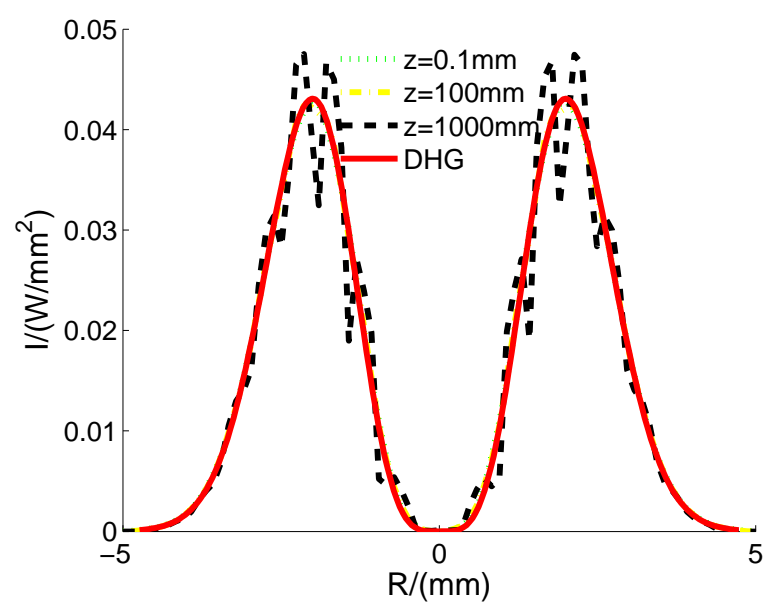

Figure 6. Ray tracing results for designs obtained with polynomial fitting: $z$ is the propagation distance from the beam shaper. After propagating only $0.1 \mathrm{~mm}$, the simulated output irradiance profile still fits well with the required DHG profile (solid line), however the irradiance profile begins to deviate from the required DHG profile with increased propagation distances.

fitting is strongly dependent on the number of piecewise polynomial functions which is defined by the number sampling points. Less sampling points reduces the simulation time significantly.

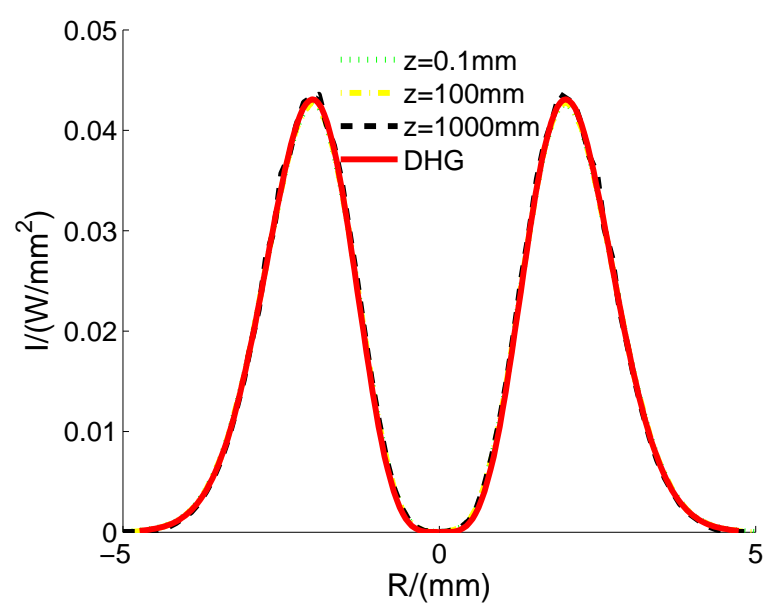

Figure 7. Ray tracing results for designs obtained with polynomial fitting: $z$ is the propagation distance from the beam shaper. The simulated irradiance profiles (dashed lines) are in excellent agreement with the required DHG profile (solid line) over the complete propagation trajectory.

\subsection{Further examples of generated complex irradiance profiles}

The presented design approach allows the design of refractive laser beam shapers for various complex irradiance profiles which are rotational-symmetric. As a further example, a Gaussian beam is transformed into an output beam with a Airy irradiance profile. Similarly as before, we used 100 points to sample the output radial coordinate $R$ and spline fitting for the surface construction. The ray tracing result with a grid of $2001 \times 2001$ rays is given in Fig. 8. The simulation result is in excellent agreement with the expected Cosine profile.

As another example, the laser beam shaper is designed to generate a Sine irradiance output profile. The output Sine irradiance profile is a Sine function shifted upwards to have zero minimum irradiance and moved 
rightwards to have a rotational symmetry axis at 0 . Applying the same design approach and simulation procedure, the ray tracing result is shown in Fig. 9. It also agrees well with the expected Sine profile.

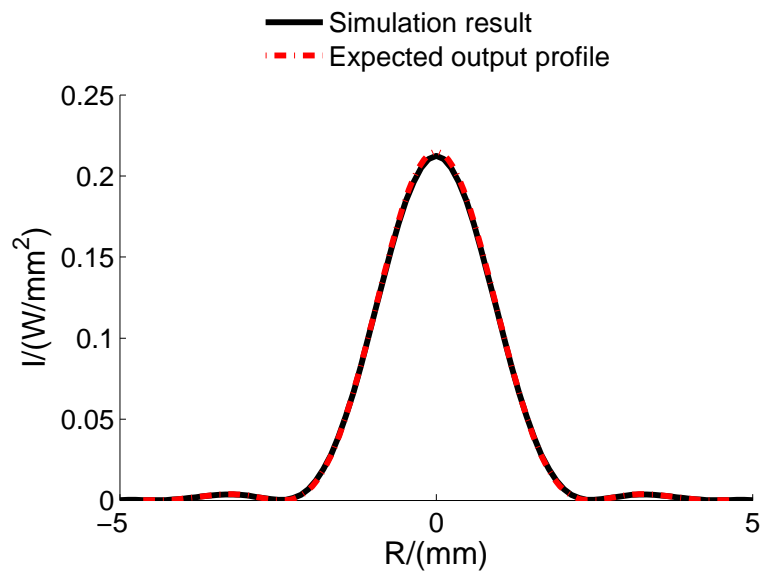

Figure 8. The output Airy irradiance profile. The simulation result (solid line) of the designed beam shaper to transform a Gaussian profile to a Airy profile shows excellent agreement with the expected output profile (dashed line).

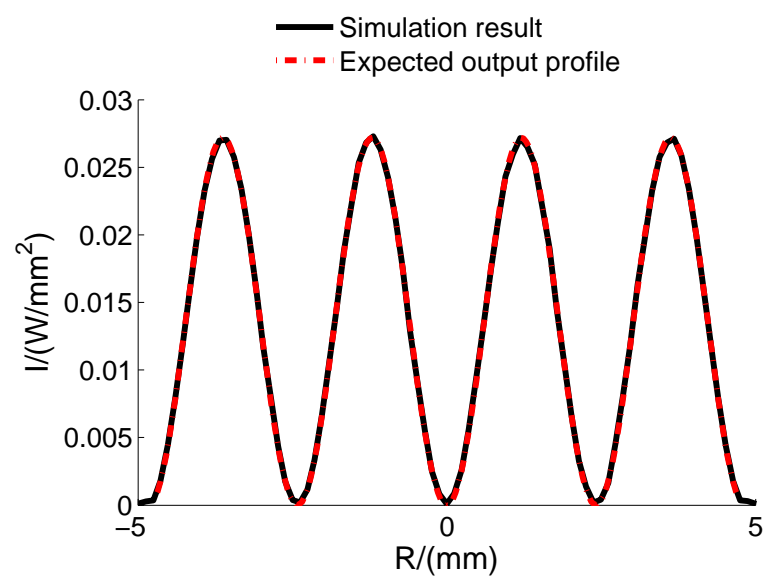

Figure 9. The output Sine irradiance profile is a Sine function shifted upwards to have zero minimum irradiance and moved rightwards to have a rotational symmetry axis at 0 . The simulation result (solid line) of the designed beam shaper to transform a Gaussian profile to a Sine profile shows excellent agreement with the expected output profile (dashed line).

These two further examples conform the versatility of our design procedure and highlight its potential use to generate different complex irradiance profiles with or without zero irradiance zones on the axis.

\section{CONCLUSION}

Ray mapping based optical design is a powerful technique to design refractive laser beam shapers for irradiance redistribution. If the desired profile is more complex than a flat-top profile, especially with low intensity in the inner region (e.g. a DHG profile), the available design procedures have to be modified. In the first step to calculate the mapping function, we have identified a "smart" sampling method. It requires much less sampling points by sampling the output radial coordinate $R$ than by sampling the encircled power $P$ conventionally. Normally only 100 sampling points work sufficiently well. In the last step to construct the calculated aspherical 
surfaces, both polynomial fitting and spline fitting can provide satisfactory results. For polynomial fitting, the polynomial series should have both even and odd exponents rather than only even exponents. Compared with polynomial fitting, spline fitting has a better precision because the generated complex profile can propagate unchanged much longer distance along the optical axis.

The described design approach in this article can be followed easily to design a refracitve lens system to transform a Gaussian irradiance pattern to a complex pattern with rotational symmetry. It should be noticed that both the input and output beams are collimated, which means they both have plane wavefronts. In future work we will develop this approach to generate output beams with not only complex irradiance patterns but also more flexible wavefronts.

\section{ACKNOWLEDGMENTS}

The work reported in this paper is mainly funded by a SBO Project grant 110070: eSHM with AM of the Agency for Innovation by Science and Technology (IWT). It is also supported by the Research Foundation - Flanders (FWO-Vlaanderen) that provides a post-doctoral grant to Fabian Duerr, and in part by the IAPBELSPO grant IAP P7-35 photonics@be, the Industrial Research Funding (IOF), FWO (G008413N), the MP1205, COST Action, the Methusalem and Hercules foundations and the OZR of the Vrije Universiteit Brussel (VUB).

\section{REFERENCES}

[1] Kuga, T., Torii, Y., Shiokawa, N., and Hirano, T., "Novel optical trap of atoms with a doughnut beam," Phys. Rev. Lett. 78(25), 4713-4716 (1997).

[2] Yin, J. and Zhu, Y., "Optical potential for atom guidance in a dark hollow laser beam," J. Opt. Soc. Am. B 15(1), 25-33 (1998).

[3] Campbell, J. P. and Deshazer, L. G., "Near fields of truncated-gaussian apertures," J. Opt. Soc. Am. 59(11), 1427-1429 (1969).

[4] Chang, S. P., Kuo, J. M., Lee, Y. P., Lu, C. M., and Ling, K. J., "Transformation of Gaussian to coherent uniform beams by Inverse-Gaussian transmittive filters," Appl. Opt. 37, 747-752 (1998).

[5] Hoffnagle, J. A. and jefferson, C. M., "Design and performance of a refractive optical system that converts a Gaussian to a flattop beam," Appl. Opt. 39, 5488-5499 (2000).

[6] Oliker, V., "Optical design of freeform two-mirror beam-shaping systems," J. Opt. Soc. Am. A 24(12), 3741-3752 (2007).

[7] Palima, D. and Glückstad, J., "Gaussian to uniform intensity shaper based on generalized phase contrast," Opt. Express 16, 1507-1516 (2008).

[8] Dresel, T., Beyerlein, M., and Schwider, J., "Design and fabrication of computer-generated beam-shaping holograms," Appl. Opt. 35, 4615-4621 (1996).

[9] Wippermann, F., Zeitner, U. D., Dannberg, P., Bräuer, A., and Sinzinger, S., "Beam homogenizers based on chirped microlens arrays," Opt. Express 15(10), 6218-6231 (2007).

[10] Romero, L. A. and Dickey, F. M., "Lossless laser beam shaping," J. Opt. Soc. Am. A 13(4), 751-760 (1996).

[11] Frieden, B. R., "Lossless conversion of a plane laser wave to a plane wave of uniform irradiance," Appl. Opt. 4(11), 1400-1403 (1965).

[12] Kreuzer, J. L., "Coherent light optical system yielding an output beam of desired intensity distrubution at a desired equiphase surface," (1969).

[13] Rhodes, P. W. and Shealy, D. L., "Refractive optical systems for irradiance redistribution of collimated radiation their design and analysis," Appl. Opt. 19(20), 3545-3553 (1980).

[14] Hoffnagle, J. A. and Jefferson, C. M., "Refractive optical system that converts a laser beam to a collimated flat-top beam," (2001).

[15] Hoffnagle, J. A. and Jefferson, C. M., "Beam shaping with a plano-aspheric lens pair," Opt. Eng. 42(11), 3090-3099 (2003).

[16] McNeil, J. A., "Design of laser beam shaping Optics - a simple algebraic method," in [Laser Resonators and Beam Control X], Kudryashov, A. V., Paxton, A. H., and Ilchenko, V. S., eds., Proc. of SPIE 6872 (2008). [doi: $10.1117 / 12.763626]$. 
[17] Ma, H., Liu, Z., Jiang, P., Xu, X., and Du, S., "Improvement of galilean refractive beam shaping system for accurately generating near-diffraction-limited flattop beam with arbitrary beam size," Opt. Express 19(14), 13105-13117 (2011).

[18] http://www. mathworks.com/.

[19] de Boor, C., [A practical guide to splines], Springer-Verlag (1978).

[20] http://www.breault.com/software/asap.php/.

[21] Cai, Y. and Lin, Q., "Hollow elliptical gaussian beam and its propagation through aligned and misaligned paraxial optical systems," J. Opt. Soc. Am. A 21(6), 1058-1065 (2004). 